\title{
RESEÑAS DE EVENTOS
}

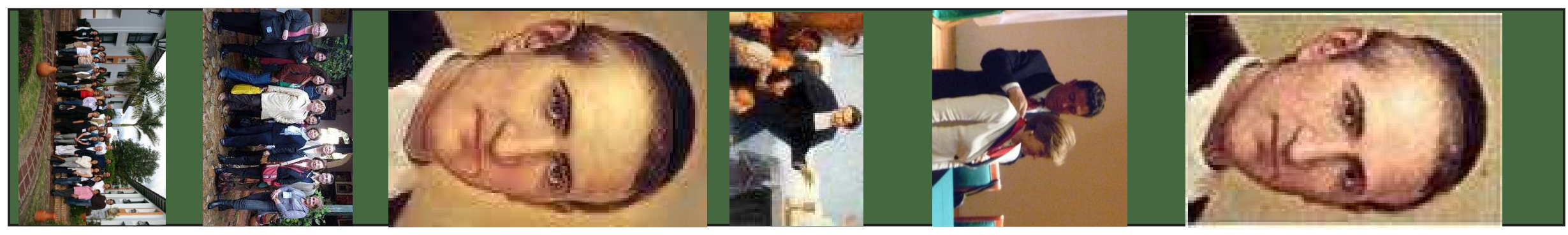




\section{PRIMER COLOQUIO NACIONAL DE LA MUJER. LIDERAZGO FEMENINO EN LA HISTORIA DE COLOMBIA}

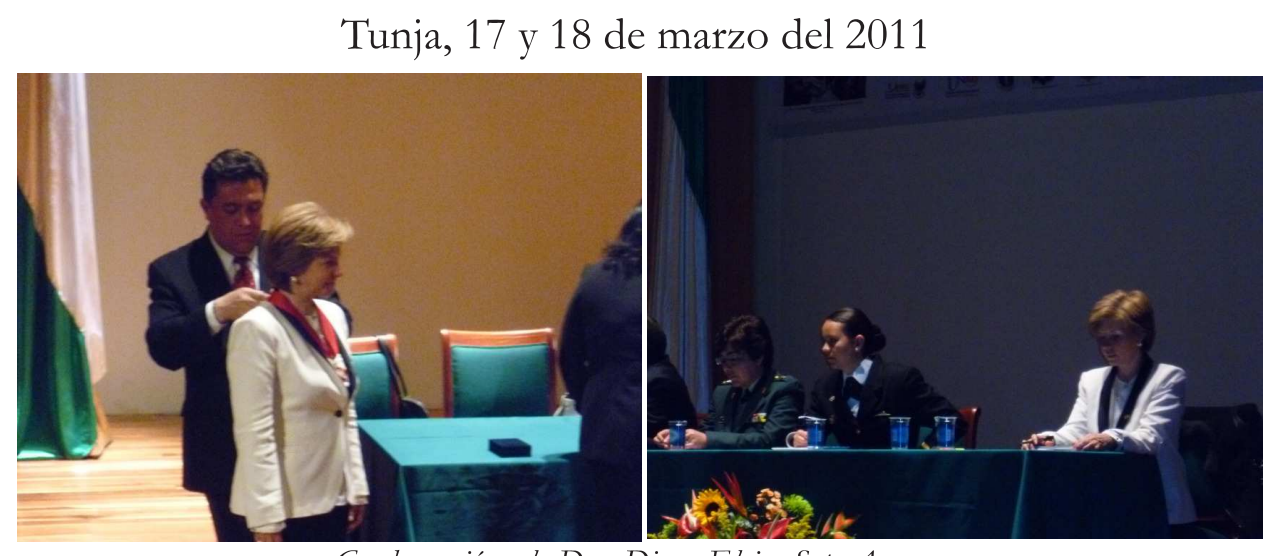

Condecoración a la Dra. Diana Elvira Soto Arango

En la ciudad de Tunja, Boyacá, se llevó a cabo el "Primer Coloquio Nacional de la Mujer. Liderazgo femenino en la historia de Colombia", durante los días 17 y 18 de marzo del 2011. El evento fue convocado por la Organización de mujeres de Boyacá, Academia Boyacense de Historia, Banco de la República, Asociación Colombiana de Historiadores, Universidad de Boyacá, CODES, Mesa Redonda Panamericana de Tunja, Universidad Pedagógica y Tecnológica de Colombia, entre otras.

La característica del evento consistió en destacar el liderazgo de la mujer colombiana en los diferentes ámbitos de la vida nacional: educación, política, economía, periodismo, fuerzas militares. Asimismo, haber conseguido reunir estas mujeres colombianas que en el siglo XX y XXI lograron liderar procesos académicos y representaciones políticas. Se destacó la presencia de la primera ministra de trabajo en Colombia; la primera periodista, las primeras doctoras, la primera mujer comandante de Barco en la armada Nacional, la primera oficial del ejército colombiano.

De gran relevancia fue la condecoración concedida a varias mujeres pertenecientes al Doctorado Ciencias de la Educación de RUDECOLOMBIA: Dra. Diana Soto Arango, Dra. Miryam Báez; Dra. Antonia Agreda. 


\title{
CENTRO DE EXCELENCIA VENDIMIA
}

\author{
Circular 05 \\ Cartagena, Bucaramanga, Ibagué, Pasto, Pereira, Tunja, \\ 23 mayo del 2011
}

\section{Presentación}

VENDIMIA, es un Centro de investigación que reúne las características de un Centro de Excelencia. Correspondió a la iniciativa de varios grupos de investigación que en su momento lideró el Grupo de Investigación: "Historia y Prospectiva de la Universidad Latinoamericana - HISULA". El presente documento resume la trayectoria académicoinvestigativa del Centro VENDIMIA que, desde marzo del 2006, se organizó bajo una convocatoria de COLCIENCIAS.

Esta red de grupos de investigación nacionales e internacionales en estos cinco años ha logrado impactar en Colombia e Iberoamérica fundamentalmente por los lineamientos del centro orientados a: Capacitación de sus miembros, investigaciones y publicaciones. Por la madurez y experiencia investigativa de la Red de grupos de investigación el Centro se encuentra en proceso de institucionalización en cada una de las instituciones participantes en el proyecto marco de investigación.

Trayectoria de VENDIMIA 2006-2011

El 30 de marzo del año 2006, los grupos de investigación fundadores de VENDIMIA, se presentaron a COLCIENCIAS a la convocatoria de Centros de Excelencia con el proyecto de investigación : "Educación,

\footnotetext{
${ }_{2}^{1}$ Véase Convenio Marco firmado por las Universidades representadas en VENDIMIA.

Diana Soto Arango, Directora de Grupo de Investigación: "Historia y prospectiva de la Universidad Latinoamericana”. HISULA. invitó a participar en la citada convocatoria a los siguientes grupos: Currículo Universitario. (Universidad de Caldas, Manizales). RUECA. Red Universitaria Evaluación de la Calidad. Colombia Universidad de Cartagena). Sociedad, Política e Historias Conectadas. (Universidad EAFIT, Medellín). Políticas Públicas y Gobernabilidad. (ESAP, Bogotá). Organizaciones y Gerencia Pública. (ESAP, Bogotá) Currículo y Universidad. (Universidad de Nariño, Pasto) Historia, Educación y Desarrollo. (Universidad de Nariño, Pasto) Historia de la Universidad Colombiana. (Universidad de Tecnológica de Pereira).

${ }^{3}$ Véase Discurso de clausura de Diana Soto Arango. Coordinadora de VENDIMIA. "Vendimia surgió como respuesta de varios grupos de investigación a la convocatoria de COLCIENCIAS número XX del 30 de mayo de 2006, para conformar "Centros de Excelencia" en Colombia. Villa de Leyva, 6 de octubre del 2007.
} 
cultura política e interculturalidad en las regiones y en los proyectos de nación en Colombia e Iberoamérica. Siglos XVIII a XXI'. Si bien el tema no fue seleccionado, por no ser prioridad para el país, los grupos integrantes decidieron continuar con la propuesta financiándola con sus propios medios. Es así que los grupos se dieron cita con el I Seminario Taller de Vendimia con el tema de "Conceptos y métodos para la bistoria y la construcción de nación", en Villa de Leyva, del 4 al 6 de octubre de 2007, bajo la coordinación de Diana Soto Arango. En este I Seminario Taller se presentó como objetivo "Capacitar a los miembros de Vendimia conceptualizando y llegando a acuerdos teórico-metodológicos sobre "Conceptos y métodos para la historia y la construcción de nación". Las discusiones y aportes generados en las distintas mesas de trabajo durante este primer seminario - taller, se concretaron en la clausura, el 6 de octubre, donde se acordó:

1. Constituir a Vendimia como "Centro de investigación de Excelencia" el cual reúne investigaciones adelantadas por los grupos que la integran, en las áreas de educación, ciencias sociales y humanas, a partir del proyecto marco definido, así: "Educación, Ciencias Sociales y Humanas" y con el Proyecto Marco: Educación, cultura política e interculturalidad en las regiones y en los proyectos de nación en Colombia. Siglos XVIII a XXI. Los grupos firmantes fueron los fundadores de VENDIMIA"

2. Avanzar en la conceptualización sobre VENDIMIA, entendiendo que la conforman, varios grupos que articulan esfuerzos académicosfinancieros para realizar una propuesta de investigación a largo plazo.

3. Mantener viva la propuesta a través de RUDECOLOMBIA bajo la temática que empezó aglutinar proyectos de tesis doctorales y de investigaciones de profesores. En este contexto, se citó y se promovió el programa de posdoctorado que fue aprobado en RUDECOLOMBIA y posteriormente en el año 2010 en la Universidad Pedagógica y Tecnológica de Colombia. Asimismo, la cohorte 15 del CADE de la Universidad Pedagógica y Tecnológica de Colombia se abrió con la línea de formación "Universidad y nación" bajo el proyecto marco de "La universidad en la construcción de nación”. Aprobado por la Dirección de Investigaciones. DIN en la Universidad Pedagógica y Tecnológica de Colombia.

Hasta ahora lo esencial en prospectiva es que se programó el " $I I$ Seminario Taller Internacional de Vendimia. 2008" sobre la temática de "La

\footnotetext{
${ }^{4}$ El evento fue coordinado en la parte académica por: Diana Soto Arango y Juan Camilo Escobar. La organización del evento estuvo a cargo del CADE de la Universidad Pedagógica y Tecnológica de Colombia bajo la coordinación la Dra. Margoth Guzmán.
} 


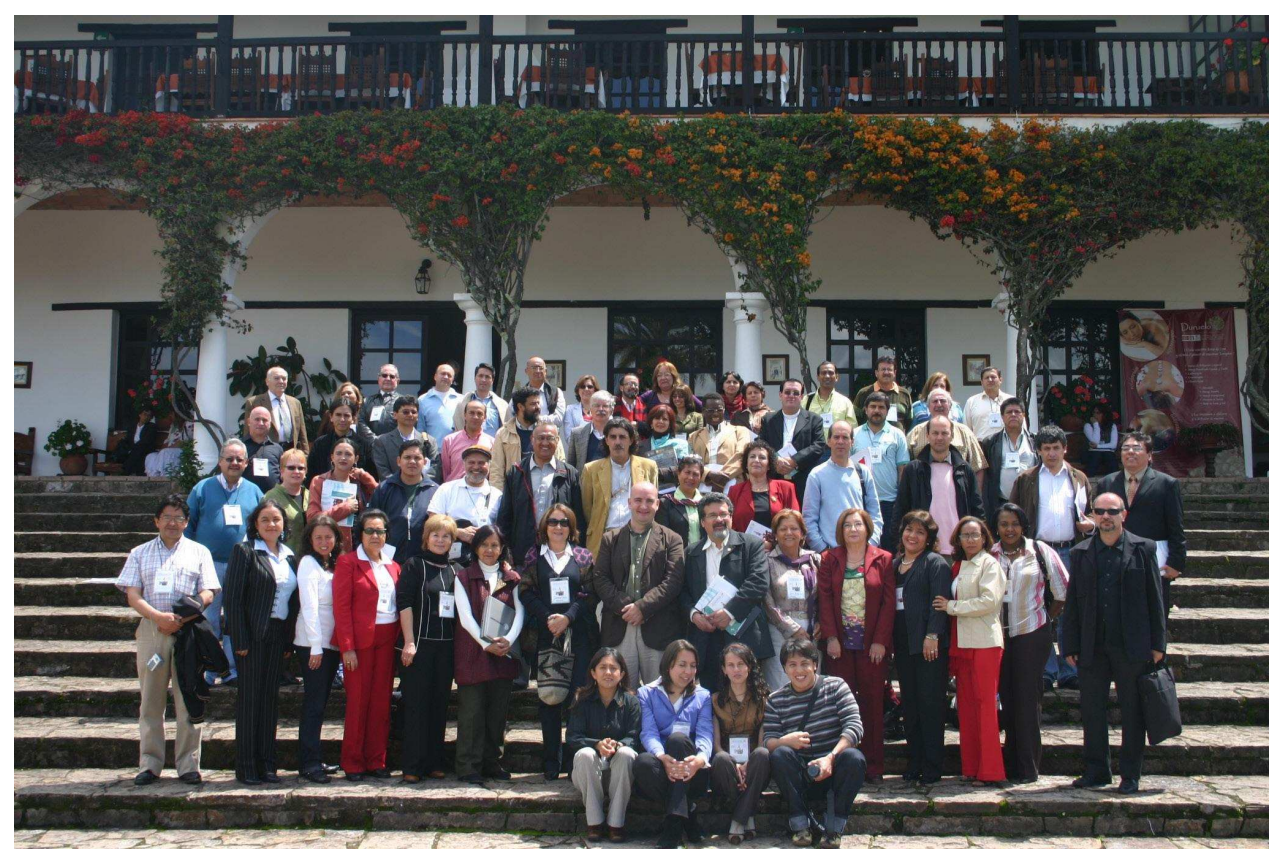

II Seminario Taller Internacional de Vendimia. 2008.

construcción de la nación en Hispanoamérica. Siglo XIX”, que se realizó en Villa de Leyva, del 12 al 14 de noviembre de 2008, bajo la coordinación de Diana Soto Arango, Dr. Juan Camilo Escobar y con el apoyo de la Dra. Margoht Guzmán, directora del CADE de la Universidad Pedagógica y Tecnológica de Colombia. En esta ocasión se dio cuenta de los avances investigativos de cada grupo y se analizó la temática propuesta con pares académicos internacionales de: Alemania, Argentina, Brasil. España, México y Venezuela.

Continuando con los avances del trabajo colectivo de VENDIMIA, se proyectó garantizar la puesta en común de los avances de investigación y la publicación de los resultados de los eventos. Por todo ello, se programo el "III Seminario Taller Internacional de Vendimia. 2009", sobre la temática de "La construcción de la nación en Latinoamérica. Siglo XIX”. El evento se realizó en Villa de Leyva, noviembre de 2009 bajo la coordinación de la Dra. Nubia Agudelo y Diana Soto Arango.

El IV Seminario Taller se desarrolló bajo la temática "Construcción de la Nación: La Universidad del Futuro en Iberoamérica". El Seminario Internacional fue coordinado por la Dra. Alba Nidia Triana y Dra. Diana Soto Arango bajo la secretaría general de la joven investigadora Carolina Tovar. En el año 2011 se desarrollará el V Seminario Taller dentro de los 15 años de RUDECOLOMBIA, del 24 al 26 de agosto en la Universidad de Santa Marta, bajo la coordinación de la Dra. Liliana del Basto, de la Universidad del Tolima. 


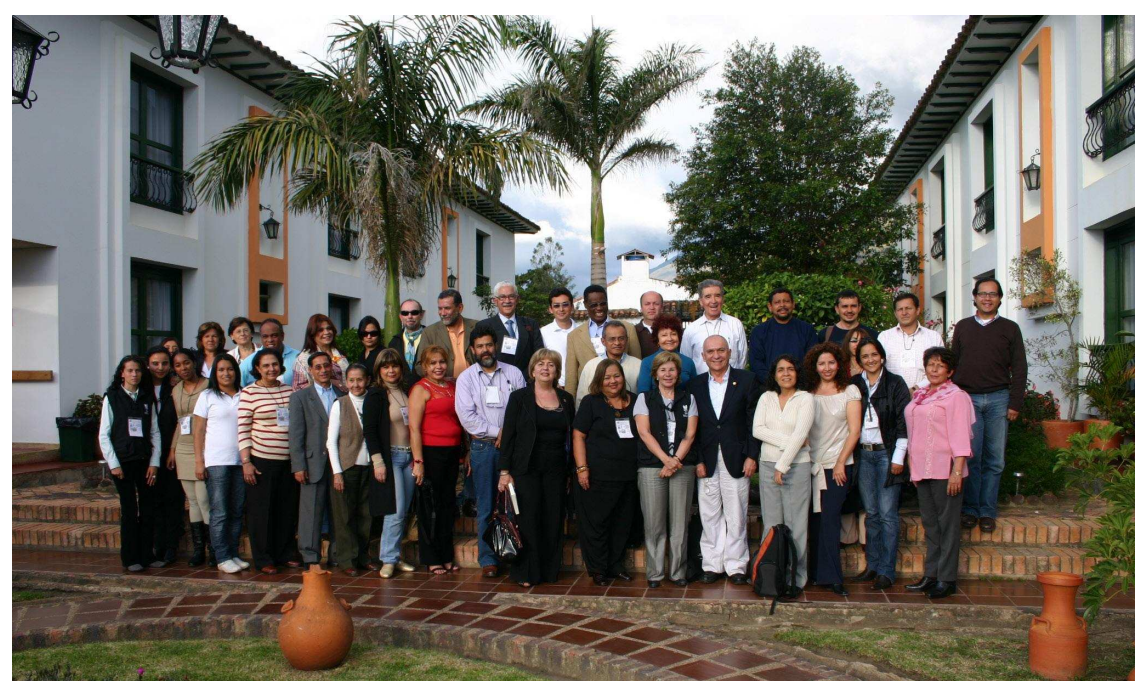

III Seminario Taller Internacional de Vendimia. 2009.

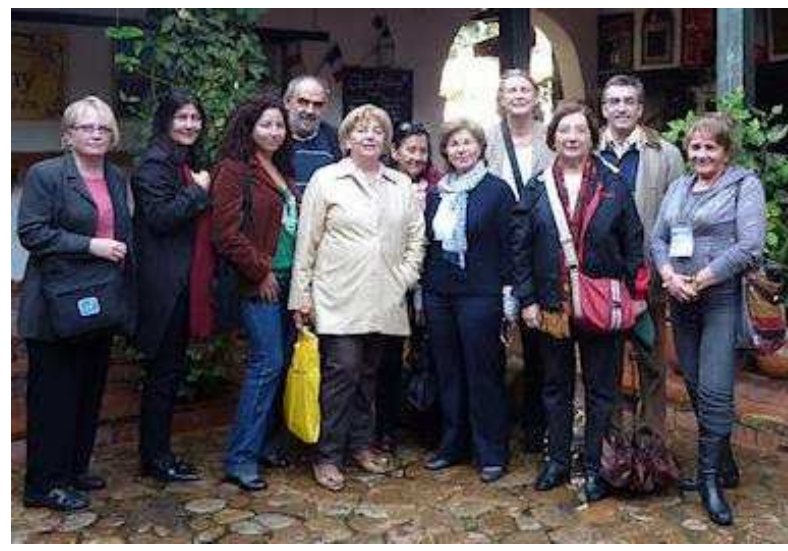

IV Seminario Taller Internacional de Vendimia. 2010.
En definitiva, podemos establecer que con estos cuatro eventos se ha dado continuidad a los objetivos centrales de VENDIMIA como son: capacitar a sus miembros y presentar los avances de los proyectos de investigación. Debemos indicar que en cada uno de los eventos se ha realizado una reunión de los directores de los grupos de investigación que conforman a VENDIMIA, donde se traza la ruta académica de cada año.

\section{Los proyectos de investigación punto de encuentro en Vendimia}

En primera instancia, debemos señalar que VENDIMIA, en este momento ya no es una propuesta para COLCIENCIAS. Si bien el desarrollo académico conceptual lo sigue dando esta institución por ser nuestro organismo directriz de la política de investigación en Colombia.

\footnotetext{
${ }^{5}$ Un Centro de Excelencia es una red nacional de grupos de investigación del más alto nivel articulada alrededor de un programa común de trabajo en un área científica y tecnológica considerada como estratégica para el país. Dentro de los centros de investigación de excelencia van a trabajar grupos de investigación de los sectores académico y productivo y de los centros de desarrollo tecnológico. Un grupo de investigación científica es un conjunto de personas que se reúnen para realizar investigación en una temática dada, formulan uno o varios problemas de su interés, trazan un plan estratégico de largo o mediano plazo y producen resultado de conocimiento e innovación sobre la temática de interés. (Memorias Encuentro Internacional de Investigadores, Colciencias. 2005).
} 
Por lo tanto, VENDIMIA es un "Centro de Investigación de Excelencia en Educación, Ciencias Sociales y humanas", conformados por grupos de investigación que están reconocidos y medidos por su producción académica en COLCIENCIAS y por grupos de investigación institucionalizados en otros países. Se debe indicar que es una nueva experiencia de trabajo en Red. Hay que decir, que la primera experiencia la realizamos con la Sociedad de Historia de la Educación Latinoamericana, SHELA (1994), que corresponde a una sociedad académica cuya participación fue de tipo personal. Es decir, la vinculación se realiza para avalar investigaciones individuales.

La segunda experiencia en red es la de RUDECOLOMBIA (1996) donde la participación es institucional para avalar un programa académico que corresponde al Doctorado en Ciencias de la Educación. La tercera experiencia en red le corresponde a VENDIMIA (2006), donde la participación se realiza por inscripción de grupos de investigación unidos por una temática general de investigación, que corresponde al proyecto marco"Educación, cultura política e interculturalidad en las regiones y en los proyectos de nación en Colombia e Iberoamérica. Siglos XVIII a XXI”. Es así, que cada grupo se inscribe a través de un proyecto de investigación relacionado con la temática general. Particularmente HISULA se inscribió en este centro de investigación con otro proyecto marco titulado "Universidad, Cultura Política y Ciudadana en los Proyectos de Nación en Colombia, siglos XVIII a XXI". En este proyecto marco se registran las tesis doctorales y de maestría al igual que los proyectos de los investigadores . Como hemos indicado la 15 cohorte del CADE de la Universidad Pedagógica y Tecnológica de Colombia se convocó bajo esta temática como línea de investigación.

Consideramos que el hecho más relevante, además de presentar los avances de investigación en los cuatro Seminarios-Talleres, se ha dado en el año 2011, cuando se unieron varios de los grupos de investigación nacionales e internacionales en la propuesta de investigación internacional que se presentó a la Unión Europea el 24 de marzo, sobre el tema "EDUACESS. POBLACIONES VULNERABLES", donde se puede destacar que la propuesta conforma un Programa Marco Interinstitucional (PMI) que tiene como finalidad establecer proyectos conjuntos para mejorar la calidad, la pertinencia y el acceso a la educación superior (ES) en América Latina (AL) de poblacionales

\footnotetext{
${ }^{6}$ Bajo esta propuesta de investigación se formaron las promociones del Doctorado Ciencias de la Educación del CADE UPTC, 5, 8, y 16. Avalados por el Proyecto de investigación la "Universidad en la formación de la Nación. Proyecto co-financiado por la Dirección de Investigaciones. DIN
} 
vulnerables: campesinos, indígenas y afroamericanos. Exclusiones sociales y educativas se definen como categorías interrelacionadas que se desprenden, de manera lógica, de las condiciones económicas y las relaciones de poder sobre grupos poblacionales en desventaja. En esta propuesta participaron los grupos de Colombia: Grupo de investigación: "Historia y Prospectiva de la Universidad Latinoamericana - HISULA", clasificado en A1, coordinado por la Dra. Diana Soto Arango y el grupo "Educación rural", clasificado en D, coordinado por la Dra. Alba Nidia Triana, liderando la propuesta desde la Universidad Pedagógica y Tecnológica de Colombia. El Grupo "Historia, Educación y Desarrollo", (Clasificado en B) coordinado por Gabriela Hernández Vega. Universidad de Nariño. El Grupo "Currículo, Universidad y Sociedad", (Clasificado en B) coordinado por la Dra. Liliana del Basto. El Grupo de investigación "Comunicación educativa", (Categoría A) Coordinado por la Dra. Olga Bedoya, Universidad Tecnológica de Pereira. Por la parte internacional, participaron las instituciones de: Universidad de $\mathrm{La}$ Habana, Cuba; Universidad de San Carlos de Guatemala; Universidad Agrícola, Honduras; Universidad de Aveiro, Portugal; Universidad Pablo de Olavide, de España.

Como es evidente, este Centro de Excelencia es dinámico en su actividad investigativa priorizando la internacionalidad del mismo.

Tampoco se debe olvidar que las investigaciones tienen impacto en los eventos pero si no se publican estos avances investigativos no se visibilizan. Conscientes de esta situación los grupos de VENDIMIA organizaron la "Colección Cuadernos de VENDIMIA". Por lo tanto, por las razones ya argumentadas, no menos importante es el proyecto editorial de VENDIMIA que recoge los trabajos de los avances de las investigaciones que se presentan en cada uno de los eventos programados. A la fecha se tiene publicados el volumen 1 y 2 que se presentó en el 2010. Actualmente se encuentra en elaboración el volumen 3 y 4.

\section{Organización administrativa}

El Centro de Excelencia VENDIMIA, presenta una Junta directiva y la respectiva reglamentación para la inscripción, permanencia y exclusión de los grupos en Vendimia. Las directrices se han establecido, desde el año 2008, que permiten cristalizar este proyecto y sus compromisos (documento que se anexa). Destacamos que el 8 de febrero del 2011 se realizó la Asamblea de los grupos de VENDIMIA quedando organizada la Junta Directiva de la siguiente manera: Dra. Liliana del Basto, (Presidenta); Dra. Gabriela Hernández (Vice-presidenta); Dra. Diana Soto Arango, (Secretaria); Dra. Alba Nidia Triana, (Tesorera); Vocales: Dra. Diana Lago; Dra. Olga Bedoya, Dr. Álvaro Acevedo. 


\section{Misión y Visión}

Por ser de relevancia destacamos la Misión de El "Centro de Excelencia VENDIMIA" desde la perspectiva del trabajo de una Red de investigación fomenta la formación de investigadores y presenta desarrollos investigativos sobre la educación, cultura política e interculturalidad en las regiones y en los proyectos de nación en Iberoamérica en los Siglos XVIII a XXI para comprender, teorizar e intervenir en los factores de las culturas socio-educativas.

La Visión, El "Centro de Excelencia VENDIMIA" en cinco años se posicionará en el contexto nacional e Iberoamericano por excelente nivel investigativo y de publicaciones que permitirá la consolidación de una red de investigación, modelo de trabajo, para la comunidad académica que trabaje en la construcción de los proyectos de nación”.

\section{Internacionalización}

Desde los inicios, con la organización del primer evento en el año 2007 se dio ingreso a investigadores y grupos internacionales. Es así, que en este tiempo han ingresado los siguientes grupos reconocidos, avalados y clasificados en cada uno de sus países:

- Grupo "Núcleo de estudios e investigaciones interdisciplinares sobre la educación superior" - NUPIES. Brasil. Coordinadora Dra. Celia Haas. Ingresó 2009.

- Grupo "La tradición clásica y humanística en España e Hispanoamérica". Subdirección General de Proyectos de Investigación. Ministerio de Educación. España. y Junta de Castilla y León. Universidad de León. Ref.: HUM2006-09045-C03-01/FILO. Coordinador: Dr. Jesús Paniagua. Grupo, ingresó en el 2007.

- Grupo "Historia y Teoría de la Profesión Docente", catastrado y certificado por el Consejo Nacional de Desarrollo Científico y Tecnológico-CNPQ, Brasil, Coordinador: José Rubens Lima Jardilino. Grupo ingresó en el 2007.

- Grupo de Investigación HUM 209. Integración histórica cultural, desarrollo y derechos humanos en América Latina", coordinado por Juan Marchena y Justo Cuño Bonito Universidad Pablo de OlavideColegio de América. Grupo ingresó en el 2007.

- Grupo "HEDURE" Coordinado por Dr. José Pascual. Universidad del Táchira, Venezuela. Grupo, ingresó en el 2008.

\section{Impacto}

Es necesario valorar que lo relevante de VENDIMIA es la aportación a la comunidad científica nacional e internacional de los avances de un proyecto de investigación de impacto regional que a 
su vez capacita en conjunto a un grupo de docentes-investigadores y doctorandos. Puede decirse que VENDIMIA cumple objetivos en tres vertientes: investigación, capacitación y formación académica de alto nivel como son los doctorados. Hay sin embargo que mencionar que VENDIMIA se ha financiado con recursos propios y el apoyo del ICETEX. En definitiva, VENDIMIA puede ser considerada sin lugar a dudas la experiencia más novedosa de impacto regional sustentada en trabajo en Red de investigadores y estudiantes de maestrías y doctorados en Colombia.

\section{Grupos de investigación de Vendimia en el 2011 Colombia}

Grupo de investigación "Historia y prospectiva de la universidad Latinoamericana. HISULA", (Clasificado en A1). Coordinado por Diana Soto Arango. Universidad Pedagógica y Tecnológica de Colombia, RUDECOLOMBIA. Fecha de ingreso a VENDIMIA: fundador desde marzo de 2006, líder de la propuesta. E-mail: dianaelvirasoto@gmail.com

Grupo "Red Universitaria Evaluación de la Calidad Capítulo Colombia RUECA", (Clasificado en B) coordinado por Dra. Diana Lago, Universidad de Cartagena, RUDECOLOMBIA. Fecha de ingreso a VENDIMIA: fundador desde marzo de 2006. E-mail: dianalago20@ yahoo.es

Grupo "Políticas, Sociabilidades y Representaciones HistóricoEducativas" coordinado por Álvaro Acevedo Tarazona, Universidad Tecnológica de Pereira, Universidad Industrial de Santander, Fecha de ingreso a VENDIMIA: fundador desde marzo de 2006. E-mail: alvaroac@une.net.co

Grupo "Historia, Educación y Desarrollo", (Clasificado en B) coordinado por Gabriela Hernández Vega. Universidad de Nariño. RUDECOLOMBIA. Fecha de ingreso a VENDIMIA: desde noviembre de 2008. E-mail: gahve2003@gmail.com

"Currículo, Universidad y Sociedad", (Clasificado en B) coordinado por Dra. Liliana del Basto. Universidad del Tolima. RUDECOLOMBIA. Fecha de ingreso a VENDIMIA, 11 de noviembre del 2009. E-mail: lilidelbasto@hotmail.com

Grupo: "Educación Rural" Clasificación en D. Coordinado por la Dra. Alba Nidia Triana, Universidad Pedagógica y Tecnológica de Colombia. Fecha de ingreso, noviembre del 2010. 
Grupo de investigación "Comunicación educativa”, (Categoría A) Coordinadora, Dra. Olga Bedoya, Universidad Tecnológica de Pereira. Ingreso 9 de febrero del 2011.

\section{Otros Países}

1. Núcleo de estudios e investigaciones interdisciplinares sobre la educación superior - NUPIES. Brasil. Coordinadora Dra. Celia Haas. Ingresó 2009.

2. Grupo "La tradición clásica y humanística en España e Hispanoamérica". Subdirección General de Proyectos de Investigación. Ministerio de Educación. España y Junta de Castilla y León. Universidad de León. Ref.: HUM2006-09045-C03-01/FILO. Coordinador: Dr. Jesús Paniagua. Grupo, ingresó en el 2007.

3. Grupo "Historia y Teoría de la Profesión Docente", catastrado y certificado por el Consejo Nacional de Desarrollo Científico y Tecnológico-CNPQ, Brasil, Coordinador: José Rubens Lima Jardilino. Grupo, ingresó en el 2007.

4. Grupo de Investigación HUM 209.'Integración histórica cultural, desarrollo y derechos humanos en América Latina", coordinado por Juan Marchena y Justo Cuño Bonito Universidad Pablo de OlavideColegio de América. Grupo, ingresó en el 2007.

5. "HEDURE" Coordinado por Dr. José Pascual. Universidad del Táchira, Venezuela. Grupo ingresó en el 2008.

\section{ANEXOS}

1. ANEXO 1. Grupos de Investigación fundadores

\begin{tabular}{|c|c|c|c|}
\hline NOMBRE & CATEGORÍA & INSTITUCIÓN & LÍDER \\
\hline $\begin{array}{c}\text { LÍDER DE VENDIMIA } \\
\text { HISULA }\end{array}$ & A 1 & $\begin{array}{c}\text { Universidad Pedagógica } \\
\text { y Tecnológica de } \\
\text { Colombia }\end{array}$ & Diana Soto Arango \\
\hline RUECA & B & $\begin{array}{l}\text { Universidad de } \\
\text { Cartagena }\end{array}$ & Diana Lago \\
\hline $\begin{array}{l}\text { CURRÍCULO UNIVERSITA- } \\
\text { RIO }\end{array}$ & A & Universidad de Caldas & $\begin{array}{l}\text { Raúl Ancízar } \\
\text { Munévar Molina }\end{array}$ \\
\hline $\begin{array}{l}\text { SOCIEDAD, POLÍTICA E } \\
\text { HISTORIAS CONECTADAS }\end{array}$ & A & $\begin{array}{l}\text { Universidad EAFIT } \\
\text { Medellín }\end{array}$ & $\begin{array}{c}\text { Juan Camilo } \\
\text { Escobar Villegas }\end{array}$ \\
\hline $\begin{array}{l}\text { ORGANIZACIONES Y GE- } \\
\text { RENCIA PÚBLICA }\end{array}$ & $\mathrm{C}$ & ESAP Bogotá & Elsy Luz Barrera \\
\hline $\begin{array}{l}\text { GRUPO "POLÍTICAS, SO- } \\
\text { CIABILIDADES Y REPRE- } \\
\text { SENTACIONES HISTÓRICO- } \\
\text { EDUCATIVAS" }\end{array}$ & A & $\begin{array}{l}\text { Universidad Tecnológica } \\
\text { de Pereira }\end{array}$ & $\begin{array}{l}\text { Álvaro Acevedo } \\
\text { Tarazona }\end{array}$ \\
\hline CULTURA Y REGIÓN & $\mathrm{C}$ & Universidad de Nariño & María Teresa Álvarez \\
\hline
\end{tabular}




\title{
XV CONGRESO DE LA FIEALC
}

\author{
Valencia, 11 al 13 de julio del 2011
}

\section{Tema Central AMÉRICA LATINA, GLOBALIDAD E INTEGRACIÓN}

El XV Congreso de la FIEALC, que se llevó a cabo en Valencia, España, del 11 al 13 de julio del 2011, fue organizado por el Área de Derecho Constitucional y el Instituto de Iberoamérica y el Mediterráneo (IBEM), de la Universidad Politécnica de Valencia. El tema con el cual se convocó a los investigadores, fue sobre, "América Latina, globalidad e integración".

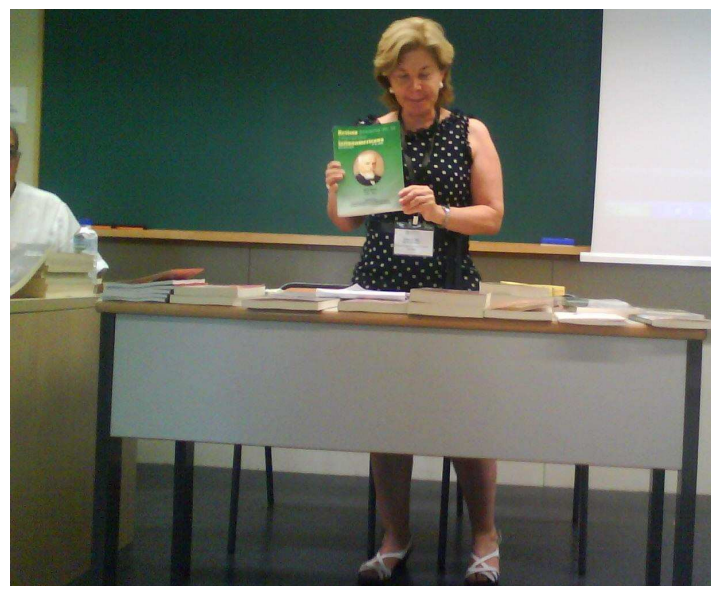

Exposición Revista Historia de la Educación Latinoamericana.
A este evento asistieron representantes de Europa, Asia, África y América, donde se presentaron 250 trabajos. En este Congreso el Grupo de investigación: "Historia y Prospectiva de la Universidad Latinoamericana HISULA", y el grupo Rueca unieron esfuerzos y organizaron la mesa de trabajo sobre "Redes Universitarias". Por parte de RUDECOLOMBIA, asistieron: Dra. Diana Soto Arango, la Directora Académica Nacional. Dra. Diana Lago, Directora CADE Universidad de Cartagena. Dra. Celina Trimiño, Directora CADE Universidad Pedagógica y Tecnológica de Colombia.

Asimismo se realizó la presentación de los libros de la Colección "Educadores Latinoamericanos" y la Revista Historia de la Educación Latinoamericana, 15. El Próximo evento se realizará en la Universidad de ANKARA en el 2013. Se promocionó el XIII CONGRESO SOLAR, que se realizará en Cartagena, (Colombia) 12, 13 y 14 de Septiembre/ 2012.

Debemos indicar que el Grupo de Investigación "Historia y Prospectiva de la Universidad Latinoamericana - HISULA", viene coordinando simposios en los congresos de la FIELAC, desde el año 2000, cuando nos vinculamos al evento de la Academia de Ciencias de Moscú.

Diana Elvira Soto Arango Universidad Pedagógica y Tecnológica de Colombia Directora grupo Investigación HISULA 


\section{ISCHE “INTERNATIONAL STANDING CONFERENCE FOR THE HISTORY OF EDUCATION" \\ San Luis de Potosí, México, 26 a 29 de julio del 2011}
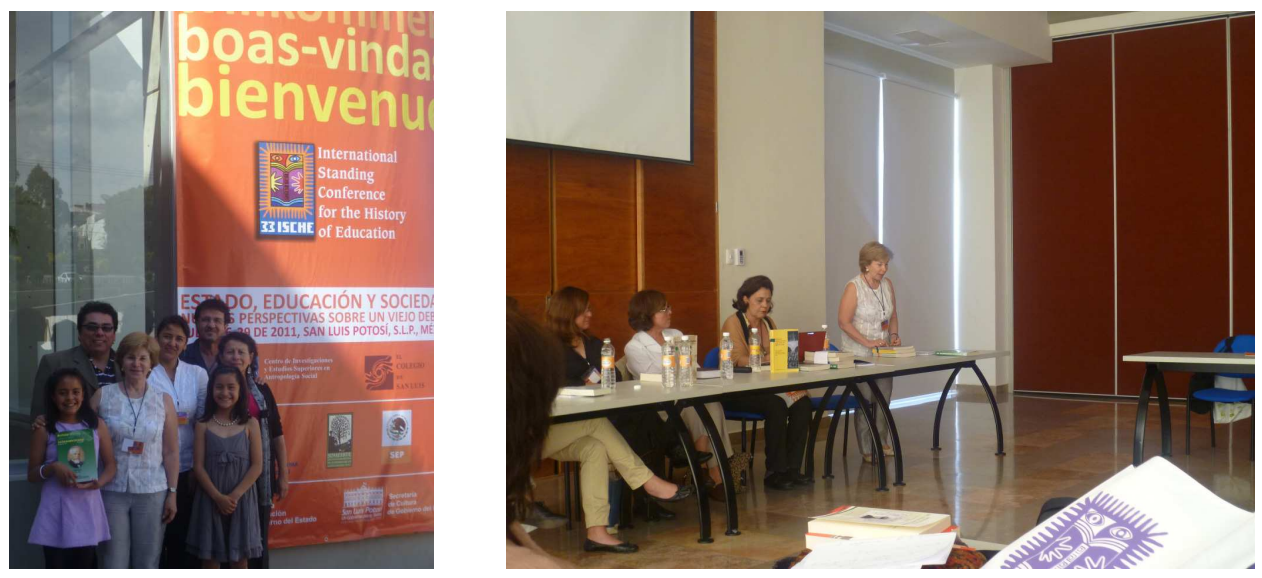

Asistentes al Evento: 33 ISCHE. International Standing Conference for the History of Education Dra. Diana Soto Arango (Colombia), Dr. José, Rubens Lima Jardilino (Brasil), Dr. Armando Martinez. Moya (México) y Dr. José Pascual Mora García (Venezuela), Dra. Doris Lilia Torres Cruz (Colombia) y Dra. Alba Nidia Trina (Colombia).

El 33 ISCHE. International Standing Conference for the History of Education, se realizó en el Colegio Mexiquense de San Luis de Potosí, México, del 26 al 29 de julio del 2011. El Congreso fue coordinado por la Dra. Luz Elena Galván. Asistieron al evento 345 investigadores de Africa, Asia, Europa, Norteamérica y de América Latina y el Caribe. El tema central giró en torno a "Estado, educación y sociedad, nuevas perspectivas sobre un viejo debate de las naciones unidas"

El Grupo de investigación "Historia y Prospectiva de la Universidad Latinoamericana- HISULA", coordinó bajo la dirección de la Dra. Therese Hamel, Dra. Diana Soto Arango, Dr. José Rubens Lima Jardilino, la Mesa de trabajo sobre "Universidad y formación de educadores". En esta mesa participaron por el Doctorado Ciencias de la Educación de la Universidad Pedagógica y Tecnológica de Colombia, RUDECOLOMBIA la Dra. Doris Torres y Dra. Alba Nidia Triana. Asimismo, en este evento se realizó la presentación de los libros de la Colección "Educadores Latinoamericanos" y la Revista Historia de la Educación Latinoamericana, 15. Se destaca que la Junta Directiva de SHELA, presente en el evento, realizó la reunión ordinaria y estableció coordinación para el ingreso de esta sociedad en la ISCHE. 
Es relevante, que durante la realización de este congreso se coordinó la elaboración de nuevas propuestas de investigación con la vinculación de investigadores de varios países bajo la coordinación de SHELAHISULA.

El próximo evento se llevará a cabo en Ginebra, Suiza en el año 2012.

Diana Elvira Soto Arango Universidad Pedagógica y Tecnológica de Colombia Directora grupo Investigación HISULA 


\title{
SOCIEDAD DE HISTORIA DE LA EDUCACIÓN LATINOAMERICANA (SHELA) SERÁ ACREDITADA POR LA INTERNATIONAL STANDING CONFERENCE FOR THE HISTORY OF EDUCATION (ISCHE)
}

\author{
33 ISCHE "INTERNATIONAL STANDING \\ CONFERENCE FOR THE HISTORY OF \\ EDUCATION"
}

San Luis de Potosí, México, 26 a 29 de julio del 2011

En el reciente Congreso 33 ISCHE realizado en San Luis de Potosí, México, entre el 26 y 29 de julio pasado, en entrevista sostenida entre la Dra. Kate Rousmaniere, President of ISCHE, y el Dr. José Pascual Mora García, Vicepresidente de SHELA, fue acordada la Carta de Intención para que la Sociedad de Historia de la Educación Latinoamericana (SHELA) obtenga su afiliación a la International Standing Conference for the History of Education (ISCHE). La delegación que asistió al 33 ISCHE estuvo integrada por los siguientes directivos de SHELA: Dra. Diana Soto Arango (Colombia), Dr. José Rubens Lima Jardilino (Brasil), Dr. Armando Martínez Moya (México), y Dr. José Pascual Mora García (Venezuela); además de los miembros de SHELA: Dra. Doris Lilia Torres Cruz (Colombia) y Dra. Alba Nidia Triana (Colombia). SHELA participó en el Standing Working Groups organizado por la Dra. Thérèse Hamel con el Simposio: Universidad $\mathrm{y}$ formación de Educadores en América.

La SHELA tiene una dilatada trayectoria que se inició en 1994 cuando fue fundada durante la celebración del "II Congreso Iberoamericano de Historiadores de la Educación Latinoamericana", realizado en UNICAMP, en la ciudad de Campignas, Brasil, del 11 al 15 de septiembre (de 1994), bajo la coordinación de Silvio Sánchez Gamboa. La Asamblea eligió como presidenta a la Dra. Diana Soto Arango (Colombia) quien fue la fundadora $\mathrm{y}$ ha sido su liderazgo fundamental para la consolidación en el tiempo de una institución de calidad y excelencia.

La Sociedad obtuvo el Registro Legal Número 145-250 de junio de 1995. Los Congresos Iberoamericanos de Historia de la Educación Latinoamericana se iniciaron teniendo como primera sede Bogotá, Colombia-1992, bajo la Coordinación de la Dra. Diana Soto Arango; Luego, Campignas, Brasil-1994 Coordinado por el Dr. Silvio Sánchez Gamboa; le siguió Caracas, Venezuela-1996, Coordinado por la Dra. Magaly Téllez; Santiago de Chile, Chile-1998, Coordinado por Don Luis Celis, quien fuera elegido Presidente; 
en Piura, Perú-2002, la mesa directiva la encabezó la Dra. María Cristina Vera de Flachs (Argentina) y fue elegida como Presidenta. En el "VI Congreso Iberoamericano de Historia de la Educación Latinoamericana", realizado en la Universidad de Guadalajara, Guadalajara-México, del 29 al 31 de octubre de 2007, la Coordinación fue del Dr. Armando Martínez Moya. En este evento en asamblea estatutaria se eligió presidente al Dr. José Rubens Lima Jardilino (Brasil). La Junta Directiva de SHELA (2007-2011) quedó integrada por el Dr. José Rubens Jardilino (Brasil), Presidente; Dr. José Pascual Mora García (Venezuela), Vicepresidente; Dr. Armando Martínez Moya (México) Tesorero; Dr. Carlos Valencia Calvo (Colombia) Tesorero; como vocales: Dra. Diana Elvira Soto Arango (Colombia), Dra. María Isabel Lafuente Guantes (España) Dra. Remedios Ferrero (España), Elmer Robles Ortiz (Perú), y la Dra. María Cristina Vera de Flachs, Expresidenta Honoraria.

Esta institución se destaca por encima de todo como una comunidad científica de primer nivel en Iberoamérica, logrando desarrollar una política editorial a través de las publicaciones como la Revista de Historia de la Educación Latinoamericana, ISSN 0122-7238, publicación arbitrada internacionalmente por investigadores activos en sus respectivos países, quienes califican además luego de un concurso público, con el mérito de estar indexada en los índices más acreditados como: LATINDEX, DIALNET, REBIUN, REDALYC, IISUE, EBSCO, CLASE, además de estar reconocida por COLCIENCIAS. Hoy la RHELA alcanza los 16 números publicados, siendo la única en su estilo en América Latina. (Cfr. www.rhela.ruecolombia.edu.co; www.shela-edu.org; www.uptc.edu.co/ doctorado/publicaciones) La SHELA publica también el Boletín de la Sociedad de Historia de la Educación Latinoamericana, que por lo general se edita previamente a los eventos en los cuales participa. Desde el punto de vista de la cultura de redes académicas en la sociedad del conocimiento ha organizado simposios, congresos y otros eventos en diferentes lugares del mundo; a manera de ejemplo podemos citar los eventos realizados en: Liverpool (Inglaterra); Moscú (Rusia); Barquisimeto (Venezuela); Macau (China); Bratislava (Eslovaquia); Tamaulipas, Guadalajara (México); Córdoba (Argentina); Varsovia (Polonia); Sevilla (España); Cartagena (Colombia); San Cristóbal (Venezuela), entre otros. Igualmente destacamos que se publican regularmente colecciones de libros, cuyos autores son principalmente miembros de la SHELA, en colaboración con otros importantes historiadores hispanoamericanos. Destacamos la reciente publicación de la colección: Educadores Latinoamericanos y del Caribe (tres tomos referidos a la colonia, siglo XIX, siglo XX y siglo XXI) ISBN: 978-958-660-178-8.

La SHELA participa en el desarrollo de la política editorial de software libre como una forma de preservar el patrimonio intelectual informático para las futuras generaciones, en un todo de acuerdo con las normativas de la Iniciativa de Budapest y la Declaración de Berlín sobre acceso abierto. En este sentido, se puede visibilizar sus publicaciones on line en los repositorios institucionales nacionales e internacionales; v. gr: www.saber.ula.ve El proyecto 
editorial Educadores Latinoamericanos está Coordinado Editorialmente por la Dra. Diana Soto Arango (Colombia), Dr. José Rubens Lima Jardilino (Brasil) y Dra. María Cristina Vera de Flachs (Argentina). Este esfuerzo editorial está patrocinado por COLCIENCIAS, Universidad Pedagógica y Tecnológica de Colombia, DIN, RUDECOLOMBIA, Universidad de LeónEspaña, Universidad Nove de Julho, Universidad Federal de Ouro Preto, UFOP, Brasil, Universidad Nacional de Córdoba, Argentina, Universidad de Cuenca, Ecuador. Universidad de Los Andes, Venezuela, Universidad de San Carlos de Guatemala, y el Grupo de investigación: "Historia y Prospectiva de la Universidad Latinoamericana" (HISULA). Con editores responsables en cada país: María Cristina Vera de Flachs - ARGENTINA; José Rubens Lima Jardilino - BRASIL; Diana Soto Arango - COLOMBIA; María Cristina Cárdenas - ECUADOR; Jesús Paniagua - ESPAÑA; Jorge Mario Rodríguez -Martínez - GUATEMAIA; José Pascual Mora García- VENEZUELA, bajo las Ediciones del Vicerrectorado Académico de la ULA.

Finalmente señalamos que la SHELA promueve las generaciones de relevo en los diferentes países Iberoamericanos a través de programas como el "Premio joven investigador Latinoamericano Diana Elvira Soto Arango". www.mineducacion.gov.co Premio aprobado por la Asamblea General de la Sociedad de Historia de la Educación Latinoamericana, en Sao Paulo, Brasil, el 24 agosto del 2009. Actualmente está abierta la convocatoria con el Tema: "Historia y prospectiva de la educación en Latinoamérica".

\section{Premios:}

1. USD 1000, para el ganador. (+) Admisión gratuita la Sociedad de Historia de la Educación (SHELA), asesoría a la dirección del proyecto y publicación del resultado en la Revista Historia de la Educación Latinoamericana.

2. Certificación; inscripción gratuita la Sociedad de Historia de la Educación (SHELA), asesoría a la dirección del proyecto y publicación del resultado en la Revista Historia de la Educación Latinoamericana.

Evaluación y criterios de evaluación: La pertinencia de la propuesta, entendida ésta como consistencia y coherencia con las líneas de investigación correspondiente a los grupos de investigación participantes en la SHELA o simposios a realizarse en el VIII Congreso de la SHELA, evento Coordinado por el Dr. Carlos Valencia Calvo en la Universidad de Caldas, entre el 29 al 31 de agosto del 2011 en Manizales, Colombia.

La delegación que asistió al 33 ISCHE Dra. Diana Soto Arango (Colombia), Dr. José Rubens Lima Jardilino (Brasil), Dr. Armando Martínez Moya (México) Dr. José Pascual Mora García (Venezuela), Dra. Doris Lilia Torres Cruz (Colombia) y Dra. Alba Nidia Trina (Colombia).

José Pascual Mora García Vicepresidente SHELA Grupo de Investigación HISULA 